\title{
ON THE DOMAIN OF NORMALITY OF AN ATTRACTIVE FIXPOINT $\left.{ }^{1}\right)$
}

\author{
BY \\ P. BHATTACHARYYA
}

\begin{abstract}
It is proved that an entire function of order less than $\frac{1}{2}$ has no unbounded immediate domains of attraction for any of its fixpoints. Estimates for the growth of functions with large infinite domains of attraction (e.g. including half planes) are obtained. It is shown that an entire function mapping an infinite domain into itself has polynomial growth in such domains.
\end{abstract}

1. Definitions. If $f(z)$ is an entire function of the complex variable $z$, then the sequence of "iterates" $\left\{f_{n}(z)\right\}$ of $f(z)$ are defined inductively by

$$
f_{0}(z)=z, \quad f_{1}(z)=f(z), \quad f_{n+1}(z)=f\left(f_{n}(z)\right), \quad n=0,1,2, \ldots,
$$

and are also entire.

If $\omega=f_{n}(z)$, then $\omega$ is called a successor of $z$ and $z$ is called a predecessor of $\omega$ in each case of order $n$.

If $f_{n}(\alpha)=\alpha$ but $f_{p}(\alpha) \neq \alpha$ for $p<n$, then $\alpha$ is called a fixpoint of order $n$ of $f(z)$. $f_{n}^{\prime}(\alpha)$ is called the multiplier of $\alpha$. Every point of the cycle $\left\{\alpha, f(\alpha), \ldots, f_{n-1}(\alpha)\right\}$ is a fixpoint of order $n$ and since $f_{n}^{\prime}(\alpha)=\prod_{k=0}^{n-1} f^{\prime}\left(f_{k}(\alpha)\right)$, every fixpoint of the cycle has the same multiplier.

A fixpoint $\alpha$ of order $n$ is called attractive, indifferent or repulsive according as $\left|f_{n}^{\prime}(\alpha)\right|<1,=1$ or $>1$ respectively. If $f_{n}^{\prime}(\alpha)=e^{2 \pi i p / q}, p, q$ integer, we say that $\alpha$ is rationally indifferent.

The set $\mathfrak{C}=\mathfrak{C}(f)$ consists of those points $z$ of the complex plane, in whose neighbourhood the sequence $\left\{f_{n}(z)\right\}$ is normal, in the sense of Montel. Its complement is denoted by $\mathscr{F}=\mathscr{F}(f)$. From the definition it is clear that $\mathfrak{C}$ is an open set, whose boundary is contained in $\mathscr{F}$.

It is easy to see that an attractive fixpoint belongs to a component of the set $\mathfrak{C}$, which is a maximal domain of normality of $\left\{f_{n}(z)\right\}$. We introduce the following definitions.

The immediate domain of attraction $D_{\alpha}$ of a first order attractive fixpoint $\alpha$ of $f(z)$ is the maximal domain of normality of $\left\{f_{n}(z)\right\}$ which contains $\alpha$. In $D_{\alpha}$, we have $\lim _{n \rightarrow \infty} f_{n}(z)=\alpha$ locally uniformly.

Received by the editors December 30, 1969 and, in revised form, April 13, 1970.

AMS 1969 subject classifications. Primary 3057; Secondary 3050.

Key words and phrases. Successor and predecessor of a point, attractive, indifferent and repulsive fixpoints, multiplier, immediate domain of attraction, domain of normality, invariant domain.

(i) Work supported by the Government of Assam, India.

Copyright (C) 1971, American Mathematical Society 
Let $\left\{\alpha_{k}\right\}$ be an attractive cycle of order $n$. Then the immediate domain of attraction $D_{\left\{\alpha_{k}\right\}}$ of the cycle is defined by $\left\{D_{\alpha_{k}}\right\}=\bigcup_{k=1}^{n} D_{\alpha_{k}}$, where $D_{\alpha_{k}}$ is the domain of normality containing $\alpha_{k}$.

The rationally indifferent fixpoints play a role, rather similar to that of attractive fixpoints. It is known [6] that a rationally indifferent fixpoint $\alpha$ belongs to the boundary of a domain of normality of $\left\{f_{n}(z)\right\}$ in which $\lim _{n \rightarrow \infty} f_{n}(z)=\alpha$ locally uniformly.

The immediate domain of attraction $D_{\alpha}$ of a rationally indifferent fixpoint $\alpha$ is the union of those maximal domains where $\left\{f_{n}(z)\right\}$ is normal and $\lim _{n \rightarrow \infty} f_{n}(z)=\alpha$, each of which has $\alpha$ as a boundary point.

2. On bounded immediate domains of attraction of an attractive fixpoint. For a polynomial, clearly the point at infinity can be considered as an attractive fixpoint. Thus for a polynomial, the immediate domain of attraction $D_{\alpha}$ of any finite attractive fixpoint $\alpha$ is bounded. This is, however, not necessarily true for entire functions. For example, if $f(z)=e^{z+a}-e^{a}, a<0$ real, then $z=0$ is an attractive fixpoint of $f(z)$. Also $\operatorname{Re} z<0$ implies $\operatorname{Re} f(z)<0$. Clearly then the left half plane $\operatorname{Re} z<0$ belongs to a domain of normality and hence to $D_{0}$, the immediate domain of attraction of 0 .

We wish to show that a result analogous to that for polynomials does, however, hold for "small" entire functions.

THEOREM 1. Let $f(z)$ be a nonconstant entire function of growth $\left(\frac{1}{2}, 0\right)$ and let $\left\{\alpha_{k}\right\}, k=1, \ldots, n$, be an attractive cycle of order $n$. Then $D_{\left\{\alpha_{k}\right\}}$, the immediate domain of attraction of the cycle $\left\{\alpha_{k}\right\}$ is bounded.

Proof. (i) We first consider the case when $\alpha_{1}=\alpha$ is a first order fixpoint. We show that in this case $D_{\alpha}$ is bounded.

Let $L$ be a continuum lying entirely in $D_{\alpha}$. Then since $f_{n}(z) \rightarrow \alpha$ locally uniformly in $D_{\alpha}$ it follows that for arbitrary $\varepsilon>0$ and for $n>n_{0}$, we have

$$
\left|f_{n}(z)-\alpha\right|<\varepsilon \text { for all } z \in L \text {. }
$$

Let $m(r)$ denote the minimum modulus of $f(z)$ on $|z|=r$. Then we have [see e.g. (5)]

$$
\lim _{r \rightarrow \infty} \sup m(r)=\infty
$$

Let $f(z)=a_{0}+a_{1} z+\cdots+a_{n} z^{n}+\cdots, a_{n} \neq 0, n>1$. Then

$$
g(z)=(f(z)-p(z)) / z^{n+1}
$$

where $p(z)=\sum_{n=0}^{n} a_{n} z^{n} ; g(z)=\sum_{r=1}^{\infty} a_{n+r} z^{r-1}$ is also of growth $\left(\frac{1}{2}, 0\right)$. From (3) we get

$$
|f(z)|>-|p(z)|+\left|z^{n+1} g(z)\right|=O\left(r^{n}\right)+r^{n+1}|g(z)|>O\left(r^{n}\right)+2 r^{n+1}
$$


for some arbitrarily large $r$ by (2). I.e. for some large $r$ we have

$$
m(r)>r^{n+1}>r .
$$

Now suppose there is a $z_{0} \in D_{\alpha}$ such that $|\alpha|<r_{1}<\left|z_{0}\right|$ where $r_{1}$ is a value for which (4) holds. There is a continuum $L$ (say an arc) lying in $D_{\alpha}$ and joining $\alpha, z_{0}$. Then the curve $f(L)$ which begins at $\alpha$ leaves $|z| \leqq r_{1}$, for there exists a $\lambda \in L$ such that $|\lambda|=r_{1},|f(\lambda)|>r_{1}^{n+1} ; n>1$ (by (4)). We now apply the same argument to the curve $f(L)$ and we see that $f_{2}(L)$ begins at $\alpha$ and leaves $|z| \leqq r_{1}$ at some point. We note that all $f_{n}(L), n=1,2,3, \ldots$, exist and are continua. By induction we can show that each $f_{n}(L)$ begins at $\alpha$ and leaves $|z| \leqq r_{1}$ at some point. Now since $r_{1}>|\alpha|$, we see that (1) is never satisfied, i.e. $L$ cannot lie in the immediate domain of attraction of $\alpha$. Hence $D_{\alpha}$ contains no points of $|z|<r_{1}$, i.e. $D_{\alpha}$ is bounded.

(ii) We now consider the cycle of fixpoints $\left\{\alpha_{1}, \ldots, \alpha_{n-1}\right\}$. We have $f_{n}\left(\alpha_{1}\right)=\alpha_{1}$, $\left|f_{n}^{\prime}\left(\alpha_{1}\right)\right|<1$ and $\alpha_{2}=f\left(\alpha_{1}\right), \ldots, \alpha_{n}=f_{n-1}\left(\alpha_{1}\right), \alpha_{n+1}=f_{n}\left(\alpha_{1}\right)=\alpha_{1}$.

Now $\alpha_{1}$ is mapped by $f(z)$ to $\alpha_{2}$ and, since $\alpha_{1}$ is an attractive fixpoint, a small neighbourhood $N$ of $\alpha_{1}$ is mapped into a small neighbourhood $f(N)$ of $\alpha_{2}$ and so on. $D_{\alpha_{k}}$ is the immediate domain of attraction of $\alpha_{k}$ as a fixpoint of $f_{n}$. Thus $f_{n m}(z) \rightarrow \alpha_{k}$ $(m \rightarrow \infty)$ in $D_{\alpha_{k}}$. It is clear that the domain of normality $D_{\alpha_{k}}$, which contains $\alpha_{k}$, is mapped by $f(z)$ to the domain of normality $D_{\alpha_{k+1}}$ containing $\alpha_{k+1}$. For suppose $z_{0}$ is any point in $D_{\alpha_{k}}$ and that $f\left(z_{0}\right) \notin D_{\alpha_{k+1}}$. Since $D_{\alpha_{k}}$ is a domain, we can join $\alpha_{k}$ to $z_{0}$ by a continuous path, say $\gamma$ in $D_{\alpha_{k}} \subset \mathbb{e}$ and $f(\gamma)$ is a connected set. Furthermore, $f(\gamma)$ joins $f\left(\alpha_{k}\right)=\alpha_{k+1}$ to $f\left(z_{0}\right)$. Now $\alpha_{k+1} \in D_{\alpha_{k+1}}$ and $f\left(z_{0}\right) \notin D_{\alpha_{k+1}}$. Hence $f(\gamma)$ must cross the boundary $\partial D_{\alpha_{k+1}}$ at least once, say at $p=f(q), q \in \gamma$. But $\gamma \subset \mathbb{E}$ and (cf. [6]) $f(\mathfrak{C}) \subset \mathfrak{C}$, so that $p=f(q) \in \mathfrak{C}$, while $q \in \partial D_{\alpha_{k+1}} \subset \mathscr{F}$ also holds, which is impossible.

Let now $R$ be so large that $|z|=R$ contains all the points $\alpha_{k}, k=1, \ldots, n$. We can choose $R_{1}>R$ suitably so as to satisfy (4).

Suppose there is a point $z_{1}$ such that $z_{1} \in D_{\alpha_{1}}$ and $\left|z_{1}\right|>R_{1}>R$. Let $L_{1}$ be a continuum (e.g. a curve) lying in $D_{\alpha_{1}}$ and joining $\alpha_{1}$ to $z_{1}$. Since $m\left(R_{1}\right)>R_{1}^{n+1}$ (by (4)), we see as in case (i) $f\left(L_{1}\right)=L_{2}$ begins at $\alpha_{2}$ and must leave $|z| \leqq R_{1}$ at some point. We thus have a path $L_{2}$ joining $\alpha_{2}$ to a point, say $z_{2}$, such that $\left|z_{2}\right|>R_{1}$.

By repeating the argument, we see that some points of $L_{3}=f\left(L_{2}\right)=f_{2}\left(L_{1}\right)$ will lie outside $|z|=R_{1}$, while also $\alpha_{3} \in L_{3}$. Similarly $f_{n}\left(L_{1}\right)$ joins $\alpha_{1}$ to some points outside $|z|=R_{1}$. Hence we see that $f_{n m}(z)(m \rightarrow \infty)$ does not tend to $\alpha_{1}$ uniformly for $z \in L_{1} \subset D_{\alpha_{1}}$. Hence $D_{\alpha_{1}}$ must be inside $|z| \leqq R_{1}$ and continuum lying in $D_{\alpha_{1}}$. Hence $D_{\alpha_{1}}$ must be inside $|z| \leqq R_{1}$ and so bounded. Similarly each $D_{\alpha_{k}}$ must be bounded, i.e. $D_{\left\{\alpha_{k}\right\}}=\bigcup_{k=1}^{n} D_{\alpha_{k}}$ is bounded.

REMARK. By exactly similar arguments, we can easily show that Theorem 1 applies to rationally indifferent fixpoints.

One can show that Theorem 1 is best possible in the sense that for any $t>0$ there exists an entire function of growth $\left(\frac{1}{2}, t\right)$ for which the immediate domain of attraction of an attractive fixpoint is unbounded. The function $f(z)=$ 
$\cos \left(\varepsilon^{2} z+9 \pi^{2} / 4\right)^{1 / 2}$ is entire and of growth $\left(\frac{1}{2}, \varepsilon\right)$ and for $0<\varepsilon<(3 \pi)^{1 / 2}, z=0$ is an attractive fixpoint. By simple computation it can be shown that the immediate domain of attraction $D_{0}$ of 0 contains the positive real axis [4].

One also has [8]

THEOREM A. For any $t>0$ there exists a function $f(z)$ of growth $\left(\frac{1}{2}, t\right)$ for which all the infinitely many first order fixpoints are attractive.

and [3]

THEOREM B. Let $\alpha$ be a first order fixpoint of $f(z)$ such that $D_{\alpha}$ is bounded. Then there exists a repulsive or rationally indifferent fixpoint of order one on the boundary $\partial D_{\alpha}$ of $D_{\alpha}$.

Theorem A and Theorem B show that Theorem 1 is best possible.

3. On functions with infinite domain of normality. In this section we consider the following problem:

Let $f(z)$ be an entire function and let $\alpha$ be a first order attractive fixpoint such that $D_{\alpha}$ is unbounded. What can we say about the growth of $|f(z)|$ as $|z| \rightarrow \infty$ in this domain of attraction?

We first consider the case when $f(z)$ is such that the immediate domain of attraction contains a half plane and $f(z)$ maps the half plane into itself. We have

THEOREM 2. Let $\alpha$ be a first order attractive fixpoint of $f(z)$ such that $\operatorname{Re} \alpha>0$ and the immediate domain of attraction $D_{\alpha}$ of $\alpha$ includes the right half plane $H: \operatorname{Re} z>0$. Further suppose $f(z)$ maps $H$ into itself. Then in any sector $|\arg z| \leqq \phi<\pi / 2,|f(z)|$ satisfies the inequality $|f(z)|<k z$, where $k>1$ is a constant which depends on $\phi$ only.

REMARK. The choice $\operatorname{Re} z>0$ for the half plane in question is clearly a trivial normalisation.

Proof. The function $w=g(z)=(z-\alpha) /(z+\alpha)$ maps $H$ one to one conformally onto the unit disc $W:|w|<1$ with $g(\alpha)=0, g(a)=-1$ where $a=(\alpha-\bar{\alpha}) / 2$. The conformal map $h(w)=g \circ f \circ g_{-1}(w)$ satisfies $h(0)=0,|h(w)|<1$ in $W$. Since

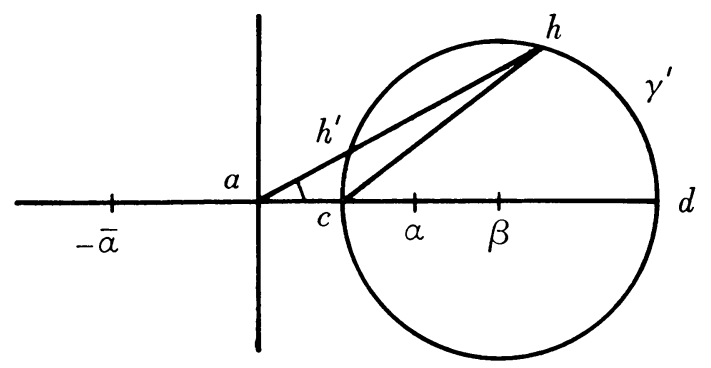

FIGURE 1 
$h^{\prime}(0)=f^{\prime}(\alpha)$ and $\left|f^{\prime}(\alpha)\right|<1, h(w)$ is not a constant multiple of $w$ and by Schwarz's Lemma we have

$$
|h(w)|<|w| \text { for all } w \in W
$$

If $\gamma$ is the circle $|w|=r<1$, then the image $\gamma^{\prime}$ under $z=g_{-1}(w)$ is a circle in $H$, whose centre $\beta$ lies on the line joining $\alpha$ with $-\bar{\alpha}$ (cf. Figure 1). By (5) the image $f\left(\gamma^{\prime}\right)$ of $\gamma^{\prime}$ is a curve lying completely within $\gamma^{\prime}$.

Let $c, d$ be the intercepts of $\gamma^{\prime}$ on the line joining $-\bar{\alpha}, \alpha$, so labelled that $c$ separates $a$ from $d$ and let the line through $a$ making an angle $\theta(<\pi / 2)$ with ad cut $\gamma^{\prime}$ in $p^{\prime}, p$ of which $p$ lies further from $a$. Set $\rho=|p-a|$. Then for $|\phi| \leqq \theta, a+\rho e^{i \phi}$ lies within $\gamma^{\prime}$ and so $f\left(a+\rho e^{i \phi}\right)$ lies within $\gamma^{\prime}$. Thus

$$
\begin{aligned}
\left|f\left(a+\rho e^{i \phi}\right)-a\right| & <|d-a| \leqq|c-a|+|d-c| \\
& =|c-a|+|p-c| / \cos \phi^{\prime} \leqq|c-a|+\rho / \cos (\theta+\psi)
\end{aligned}
$$

where $\phi^{\prime}$ is the angle $c p$ makes with $c d$ and $\psi$ is the angle between $p a$ and $p c$.

Now $c$ corresponds to $w=-r$ and $d$ to $w=r$, so that

$$
c=(-\bar{\alpha} r+\alpha) /(r+1), \quad|c-\alpha|=\left(\frac{1-r}{1+r}\right) \frac{(\alpha+\bar{\alpha})}{2} \rightarrow 0 \quad \text { as } r \rightarrow 1,
$$

and $d=(\bar{\alpha} r+\alpha) /(1-r) \rightarrow \infty$ as $r \rightarrow 1$.

Thus, if we keep $\theta$ fixed, and let $r \rightarrow 1$ we find $|c-a| \rightarrow 0, \psi \rightarrow 0, \rho \rightarrow \infty$ and so

$$
\left|f\left(a+\rho e^{i \phi}\right)\right| \leqq|a|+|c-a|+\rho / \cos (\theta+\psi)<K^{\prime} \rho
$$

uniformly in $|\phi| \leqq \theta$, as $\rho \rightarrow \infty$, where $K^{\prime}$ is any constant $>1 / \cos \theta$.

If we now consider $z$ in an angle $A$ : $|\arg z|<\phi<\pi / 2$ and take any constant $K>1 / \cos \phi$, then we may take an angle $\theta$ satisfying

$$
\begin{gathered}
\phi<\theta<\frac{\pi}{2}, \\
K>K^{\prime}>1 / \cos \theta .
\end{gathered}
$$

All but a finite part of $A$ is contained in the angle $B:|\arg (z-a)|<\theta$. Then as $z \rightarrow \infty$ in $A$ (and hence in $B$ ):

$$
\begin{aligned}
|f(z)| & =|f(a+z-a)| \\
& \leqq K^{\prime}|z-a| \quad \text { by the first part of the proof, } \\
& \leqq K|z| \quad \text { for large }|z| .
\end{aligned}
$$

This proves the theorem.

REMARK 1. It is now possible to improve this result a little further. E. Landau and G. Valiron [7] have proved the following result:

Theorem C (LANDAU-VAliRon). Let $\phi(z)$ be regular and $\operatorname{Re} \phi(z) \geqq 0$ for $\xi=\operatorname{Re} z>0$. Then we can find $c \geqq 0$, so that writing $\phi(z)-c z=\psi(z)$,

(i) $\operatorname{Re} \psi(z) \geqq 0$ for $\xi>0$,

(ii) for every $p>0, \psi(z) / z \rightarrow 0$ uniformly in $|z| \leqq p \xi$, as $|z| \rightarrow \infty$. 
This shows that the constant $K$ in Theorem 2 is the same for any angle $\theta<\pi / 2$. Letting $z \rightarrow \infty$ along the $+v e$ real axis, we can assume $\theta=0$ and from (6) we see that in Theorem 2 we can take any $K>1$.

REMARK 2. We now construct an example to show the sharpness of Theorem 2. Consider the function

$$
f(z)=e^{a+z}-e^{a}+\lambda z,
$$

where $a<0$ is real, $0<\lambda<1-e^{a}$.

Then $f(0)=0$ and $0<f^{\prime}(0)<1$, i.e. $z=0$ is an attractive fixpoint of $f(z)$.

For $\operatorname{Re} z=x$, we have

$$
f^{\prime}(x)=e^{a+x}+\lambda>0, \quad f^{\prime \prime}(x)=e^{a+x}>0 .
$$

Then $f(x)=x$ has only two roots, viz. $x=0$ and $x=c(>0)$. For $0<x<c$ we have $f(x)<c$. For any $z$ with $\operatorname{Re} z<0$, we have

$$
\begin{aligned}
\operatorname{Re} f(z) & =\operatorname{Re}\left\{e^{a+z}-e^{a}+\lambda z\right\}<\left|e^{a+z}\right|-e^{a}+\lambda c \\
& \leqq e^{a+c}-e^{a}+\lambda c=f(c)=c .
\end{aligned}
$$

Thus the half plane $H: \operatorname{Re} z<c$ is invariant, i.e. $f(H) \subset H$, and since $z=0$ is an attractive fixpoint, $H$ belongs to the immediate domain of attraction of 0 . In $H$ we have by Theorem 2

$$
|f(z)|=O(|z|)
$$

But $f(z) \sim \lambda|z|, \lambda>0$ as $z \rightarrow \infty$ in $H$ in such a way that $\operatorname{Re} z \rightarrow-\infty$.

We note that by taking $-a$ very large, we get $e^{a} \sim 0$ and so $\lambda$ may be taken arbitrarily close to 1 .

In Theorem 2 we assumed that $f(H) \subset H$. We now relax this condition and examine what we can still say about the growth of the function.

THEOREM 3. Let $\alpha$ be an attractive fixpoint of order 1 of $f(z)$ and let $\alpha$ belong to the right half plane $H: \operatorname{Re} z>0$. Let $D_{\alpha}$ the immediate domain of attraction $\alpha$ contain $H$. Then for any $\varepsilon>0, f(z)$ satisfies the inequality $|f(z)|<\lambda+\mu|z|^{2}$ in $|\arg z|<\pi / 2-\varepsilon$, where $\lambda$ and $\mu$ are constants.

(Note: We no longer assume that $f(H) \subset H$. It is, however, necessarily true that $\left.\left(D_{\alpha}\right) \subset D_{\alpha}.\right)$

Proof. Let $w=g(z)$ map $D_{\alpha}$ conformally and univalently onto the right half plane $H: \operatorname{Re} z>0$ such that $g(\alpha)=\alpha$. The function $u=h(w)=(w-\alpha) /(w+\bar{\alpha})$ maps $H$ conformally and univalently onto the unit disc $U=\{u:|u|<1\}$ with $h(\alpha)=0$.

Consider the function

$$
\tilde{\phi}(u)=h \circ g \circ f \circ g_{-1} \circ h_{-1}(u) .
$$

Clearly

$$
\delta(0)=0, \quad\left|\tilde{\phi}^{\prime}(0)\right|=\left|f^{\prime}(\alpha)\right|<1,
$$


and $\tilde{\phi}$ maps the unit disc $U$ into itself since $f$ maps $D_{\alpha}$ into itself, i.e. $|\tilde{\phi}(u)|<1$ for $|u|<1$. Hence by Schwarz's Lemma, we obtain

$$
|\tilde{\phi}(u)| \leqq|u| \text {. }
$$

Consider the circle $\gamma:|u|=r<1$. Because of (11) we know that $\gamma$ is mapped by $\tilde{\phi}(u)$ into a curve $\tilde{\gamma}$, which lies completely within or on $\gamma$ and the interior of $\gamma$ is mapped into a subset of the interior of $\gamma$.

We look at the corresponding picture in the $w$-plane and in the $z$-plane.

Now $h_{-1}(u)$ maps $\gamma$ to a circle $\gamma^{\prime}=h_{-1}(\gamma)$ in the $w$-plane, whose centre is some point $c$ lying on the line joining the two points $\alpha$ and $-\bar{\alpha}$, and $g_{-1}$ maps this circle to some Jordan curve $\gamma^{\prime \prime}=g_{-1} \circ h_{-1}(\gamma)$ in the $z$-plane (cf. Figure 2). Further, the interior of $\gamma$ is mapped univalently onto the interior of $\gamma^{\prime \prime}$ by $g_{-1} \circ h_{-1}$.

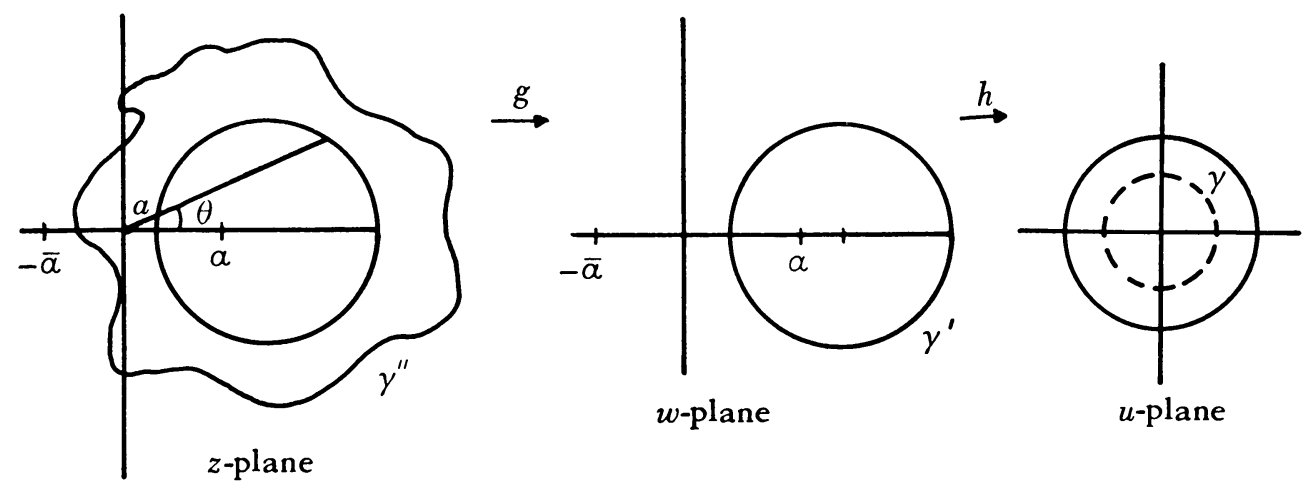

FIGURE 2

Since $\bar{\phi}=h \circ g \circ f \circ g_{-1} \circ h_{-1}$ maps the interior of $\gamma$ into a subset of its own interior, it is clear that $f$ must map $\gamma^{\prime \prime}$ into some curve lying completely within $\gamma^{\prime \prime}$. Thus the interior $\Delta$ of $\gamma^{\prime \prime}$ is mapped by $f$ into a bounded domain, whose boundary lies within $\gamma^{\prime \prime}$, the boundary of $\Delta$. Hence

$$
f(\Delta) \subset \Delta .
$$

Now $\gamma^{\prime \prime}$ is the image of $\gamma$ under the one to one conformal map

$$
z=\psi(u)=g_{-1} \circ h_{-1}(u)
$$

which maps the unit disc $U=\{u:|u|<1\}$ one to one onto the immediate domain of attraction $D_{\alpha}$ of $\alpha$ and carries the origin $u=0$ to $\alpha$. Let $\psi^{\prime}(0)=\lambda(\neq 0)$. Then the function

$$
\tilde{\psi}=(\psi-\alpha) / \lambda=u+\cdots
$$

is univalent in $|u|<1$ and normalised. Applying Koebe's distortion formula to (13) we obtain

$$
|(\psi(u)-\alpha) / \lambda| \leqq r /(1-r)^{2} \text { for }|u| \leqq r \leqq 1,
$$


and hence

$$
|\psi(u)| \leqq|\alpha|+|\lambda| /(1-r)^{2} \text { for }|u| \leqq r<1 .
$$

Let $a=(\alpha-\bar{\alpha}) / 2$ and let $c, d, p^{\prime}, p, \rho$ be defined as in the proof of Theorem 2 (see Figure 1) where $\gamma^{\prime}$ has the same meaning as in our present theorem. Now the circle $\gamma^{\prime}$ lies inside $\gamma^{\prime \prime}=g_{-1} \circ\left(h_{-1}(\gamma)\right)$. For $G(u)=h \circ g \circ g \circ g_{-1} \circ h_{-1}(u)$ maps $U=$ $\{u:|u|<1\}$ into a proper subset of itself and $G(0)=0$. Thus $|G(u)|<|u|$ for $|u|<1$ by Schwarz's Lemma. Thus $G(\gamma)=h \circ g \circ g \circ g_{-1} \circ h_{-1}(\gamma)$ lies inside $\gamma$, i.e. $g \circ g_{-1} \circ h_{-1}(\gamma)$ lies inside $g_{-1} \circ h_{-1}(\gamma)$, i.e. $h_{-1}(\gamma)$ lies inside $\gamma^{\prime \prime}$.

Then, since for $|\phi|<\theta, a+\rho e^{i \phi}$ is inside $\gamma^{\prime \prime}$, we have $f\left(a+\rho e^{i \phi}\right)$ inside $\gamma^{\prime \prime}$ and so by (14),

$$
\left|f\left(a+\rho e^{i \phi}\right)\right|<|\alpha|+|\lambda| /\left(1-r^{2}\right) .
$$

We now estimate $\rho$ from

$$
u=h(w)=(w-\alpha) /(w+\alpha), \quad w=(\alpha+u \bar{a}) /(1-u),
$$

whence

$$
\left|w-\left(\frac{\alpha-\bar{\alpha}}{2}\right)\right|=\frac{a+\bar{\alpha}}{2}\left|\frac{1+u}{1-u}\right| .
$$

Putting $u=r e^{i \beta}, E=(\alpha+\bar{\alpha}) / 2$ we see that the maximum of the above expression occurs when $\beta=0, w=d$, i.e. [Figure 3]

$$
|d-a|=E(1+r) /(1-r) \text {. }
$$

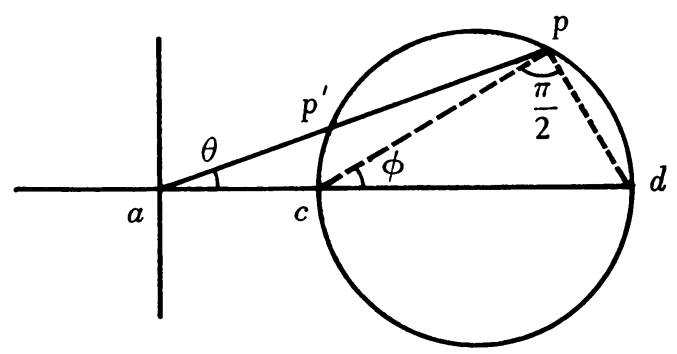

FIGURE 3

Now $|p-c|=|d-c| \cos \phi$. If now we let $r \rightarrow 1$, i.e. $p \rightarrow \infty$ and (cf. Theorem 2)

$$
|c-a| \rightarrow 0, \quad|p-c|=a p+o(1)=\rho+o(1) .
$$

But

$$
\begin{aligned}
|p-c| & =|d-c| \cos \phi=\{|d-a|+o(1)\} \cos \phi \\
& =E((1+r) /(1-r)) \cos \theta+o(1) \quad \text { as } r \rightarrow 1 .
\end{aligned}
$$

Thus as $r \rightarrow 1, \rho \rightarrow \infty$ and in fact

$$
\rho=E((1+r) /(1-r)) \cos \theta+o(1)=2 E(1+o(1)) \cos \theta /(1-r) .
$$


Thus for suitable constants $E^{\prime}, \lambda^{\prime}$ and $|\phi| \leqq \theta<\pi / 2$,

$$
\left|f\left(a+\rho e^{i \phi}\right)\right|<|\alpha|+\frac{\lambda^{\prime}}{E^{\prime 2} \cos ^{2} \theta} \rho^{2}(1+o(1)),
$$

i.e. $\left|f\left(a+\rho e^{i \phi}\right)\right|<A+\lambda^{\prime \prime} \rho^{2}$ uniformly in $\phi$ for a suitable constant $\lambda^{\prime \prime}$ (depending on $\theta)$. Transforming to the point $z$ we get

$$
|f(z)|<\lambda+\mu|z|^{2} \quad \text { in }|\arg z|<\pi / 2-\varepsilon
$$

by the same argument as at the end of Theorem 2 .

The proof of Theorem 3 is now complete.

We note that in the above theorem we needed the attractive fixpoint $a$ to be in the right half plane $\operatorname{Re} z>0$. In view of the fact that $D_{\alpha}$ may well contain parts of the plane $\operatorname{Re} z<0$, this assumption is rather strong. However, we can go to more general situations by quite simple conformal mapping arguments. Denote by an invariant domain a domain $D$ such that $f(D) \subset D$. The $D_{\alpha}$, belonging to an attractive fixpoint $\alpha$, is invariant in this sense. We prove

THEOREM 4. (i) Let $D$ be an infinite simply connected invariant domain of an entire function (not necessarily containing an attractive fixpoint).

(ii) Let $D$ contain an angle $A$ : $\alpha<\arg \left(z-z_{0}\right)<\alpha+\beta, \beta>0$. Then for arbitrary $\varepsilon>0$,

$$
|f(z)|=O\left(|z|^{k}\right) \text { for some } k=k(\beta, \varepsilon)
$$

as $|z| \rightarrow \infty$ in $\alpha+\varepsilon \leqq \arg \left(z-z_{0}\right)<\alpha+\beta-\varepsilon$.

Proof. Without loss of generality, we can assume $\alpha=-\beta / 2$. Let $z_{1}$ be any finite boundary point of $D$. Then the ray $z=z_{1}+x, x \geqq 0$, meets the boundary of $D$ a last time (i.e. for maximum $x$ ) at say $z_{2}$ and meets the boundary of $A$ at say $z_{3}$. Denote $A_{1}(\subset A)$ the sector $\left\{z \mid-\beta / 2<\arg \left(z-z_{3}\right)<\beta / 2\right\}$. Now $z-z_{3}=t^{\beta / \pi}$ maps the right half plane $\operatorname{Re} t>0$ onto $A_{1}$. Further there is a branch $\psi(z)$ of $\left(z-z_{2}\right)^{1 / 4}$ regular and single valued in $D$ (by the Monodromy Theorem) and taking real positive values on $L: \arg \left(z-z_{2}\right)=0$. Then $w=\psi(z)$ maps $D$ into the half plane $\operatorname{Re} w>0$. [For the image $\psi(D)$ contains the $+v e$ real $w$ axis and if it contains say $w^{\prime}$ in $\operatorname{Re} w<0$, then $w^{\prime}$ can be joined by $w=1$ by a path which meets $\operatorname{Re} w=0$ a last time at say $w^{\prime \prime}$ (say) and after that meets the positive real axis for the first time at $w^{\prime \prime \prime}$.

The segment $w^{\prime \prime \prime} w^{\prime \prime}$ of the path lies in $\psi(D)$ and is mapped by $z-z_{2}=w^{4}$ into a curve (in $D$ ) joining

$$
z^{m}=z_{2}+\left|w^{m}\right|^{4} \text { and } z^{\prime \prime}=z_{2}+\left|w^{\prime \prime}\right|^{4}
$$

on which $\arg z$ changes by $2 \pi$. This curve together with the real segment $z^{\prime \prime} z^{\prime \prime \prime}$ (which is also in $D$ ) separates $z_{2}$ from distant points of the boundary of $D$, against the fact that the boundary cannot contain a finite isolated component.]

Now $z_{3}+t^{\beta / \pi}$ maps $t$ in the right half plane $\operatorname{Re} t>0$ to a value in $A_{1}(\subset D)$ and further $f\left(z_{3}+t^{\beta / \pi}\right)$ belongs to $D$ and $\phi(t)=\psi\left(f\left(z_{3}+t^{\beta / \pi}\right)\right)$ belongs to the right half 
plane. Hence, $|\phi(t)|=O(|t|)$ in any angle $|\arg t| \leqq \pi / 2-\delta$, by the Landau-Valiron result (Theorem $\mathrm{C}$ ). Thus,

$$
\left|f\left(z_{3}+t^{\beta / \pi}\right)-z_{2}\right|=O\left(|t|^{4}\right), \quad\left|f\left(z_{3}+t^{\beta / \pi}\right)\right|=O\left(|t|^{4}\right)
$$

as $t \rightarrow \infty$ in $|\arg t| \leqq \pi / 2-\delta$, i.e. $|f(z)|=O\left(|z|^{4 \pi / \beta}\right)\left(\right.$ since $\left.O\left(\left|z-z_{3}\right|^{4 \pi / \beta}\right) \simeq O\left(|z|^{4 \pi / \beta}\right)\right)$ as $z \rightarrow \infty$ in any angle of the form $\left|\arg \left(z-z_{3}\right)\right|<\beta / 2-\varepsilon$.

This completes the proof of Theorem 4.

Using Phragmen-Lindelöf methods and our growth estimate in Theorem 4, one sees that functions having invariant domains including an infinite angular sector must have a certain minimal order of growth. One has (see e.g. [1])

LEMMA. If the order of the entire function $f(z)$ is $\langle\gamma, \gamma\rangle 0$ and if as $z \rightarrow \infty$ outside a number of disjoint angular sectors of the form $D$ :

$$
\theta_{1}<\arg z<\theta_{2}, \quad \theta_{2}-\theta_{1}<\pi / \gamma,
$$

one has $|f(z)|=O\left(\exp \left(|z|^{\gamma^{\prime}}\right)\right), \gamma^{\prime}<\gamma$. Then the order of $f(z)$ is in fact $\leqq \gamma^{\prime}$.

Application to Theorem 4. We have in Theorem 4 that $\theta_{2}-\theta_{1}$ is $2 \pi-\beta+\varepsilon$ outside of such an angle, i.e. in a slightly smaller sector than $A$, we have $f(z)$ of polynomial growth, so that $\gamma^{\prime}$ may be taken arbitrarily small. $\gamma$ may be taken as any number $<\pi /(2 \pi-\beta+\varepsilon)$. We see then that the order of an $f(z)$ possessing such an invariant region as in Theorem 4 is at least $\pi /(2 \pi-\beta)$. That this is sharp for $\beta=\pi$ is shown by the case of $f(z)=e^{z}-1$ [2].

I am indebted to Dr. I. N. Baker of Imperial College, London, for his help and suggestions in preparing this paper.

\section{REFERENCES}

1. I. N. Baker, Entire functions with linearly distributed values, Math. Z. 86 (1964), 263-267. MR 30 \#3977.

2. P. Bhattacharyya, On the set of normality and iteration of $e^{z}-1$, Acta. Sci. Math. (Szeged) (to appear).

3. - On Boundaries of domains of attraction, Publ. Math. Debrecen (to appear).

4. - Iteration of analytic functions, Ph.D. Thesis, University of London, 1969.

5. R. Boas, Jr., Entire functions, Academic Press, New York, 1954. MR 16, 914.

6. P. Fatou, Sur les équations functionelles, Bull. Soc. Math. France 47 (1919), 161-271.

7. E. Landau and G. Valiron, A deduction from Schwarz's Lemma, J. London Math. Soc. 4 (1929), 162-163.

8. J. E. Whittington, On the fixpoints of entire functions, Proc. London Math. Soc. (3) 17 (1967), 530-546. MR $35 \# 5616$.

UNIVERSITY OF MINNESOTA, MinNEAPOLIS, MinNesota 55455 